\title{
Ketamine Infusion (KI) in Treatment Resistant Depression (TRD): Retrospective Clinical Data Analysis
}

\author{
Chris Griffiths' ${ }^{1}$, Alex 0’Neill-Kerr¹, Robert De Vai' ${ }^{1}$, Tina Sore'1, Ksenija Da Silva ${ }^{2}$ \\ ${ }^{1}$ Innovation and Research department, Northamptonshire Healthcare NHS Foundation Trust, Northampton, UK \\ ${ }^{2}$ Faculty of Health and Society, University of Northampton, Northampton, UK \\ Email: chris.griffiths@nhft.nhs.uk
}

How to cite this paper: Griffiths, C., O'Neill-Kerr, A., De Vai, R., Sore, T., \& Da Silva, K. (2019). Ketamine Infusion (KI) in Treatment Resistant Depression (TRD): Retrospective Clinical Data Analysis. Open Journal of Depression, 8, 59-70. https://doi.org/10.4236/ojd.2019.83007

Received: July 10, 2019

Accepted: August 27, 2019

Published: August 30, 2019

Copyright $\odot 2019$ by author(s) and Scientific Research Publishing Inc. This work is licensed under the Creative Commons Attribution International License (CC BY 4.0).

http://creativecommons.org/licenses/by/4.0/

\begin{abstract}
Objective: The aim of this paper is to present the clinical data analysis results from a service delivering ketamine infusion, repetitive transcranial magnetic stimulation (rTMS) and electroconvulsive therapy for people with treatment resistant depression (TRD). Methods: The study was a retrospective investigation of routinely collected data on patients receiving ketamine infusion between 2017 and 2019. Measures used were the clinician-rated Clinical Global Impression (CGI) and self-reported Beck Depression Inventory (BDI) and Quick Inventory of Depressive Symptomatology (QIDS-SR). The outcome data of 28 patients with TRD were analysed. A minimum course of treatment was defined as three ketamine infusions. Results: Response combined with partial response, response, and remission rates respectively were $42.3 \%, 23.1 \%$ and $4.2 \%$ for the BDI; $18.2 \%, 9.1 \%$ and $0 \%$ for the QIDS-SR; $50 \%, 18.2 \%$ and $4.6 \%$ for the CGI. There was a statistically significant improvement on the BDI with a medium effect size (0.63). There was not a significant improvement in the CGI or QIDS-SR. Conclusions: The results show a mixed picture the impact ketamine on symptoms of depression in TRD over the course of multiple infusions. The results need to be viewed in the context of clinical outcome data collection: outcome scores are taken prior to treatment and so are at a point when ketamine is not having an active effect. The findings can inform the availability of ketamine infusion as a treatment option for TRD where treatment adhering to national guidelines has not succeeded. A qualitative investigation of the experience of people receiving ketamine would provide further insights.
\end{abstract}

\section{Keywords}

Ketamine Infusion, Repetitive Transcranial Magnetic Stimulation (rTMS), 


\section{Introduction}

Treatment resistant depression (TRD) does not have a single universally accepted definition (Berlim \& Turecki, 2007). Many people experiencing major depressive disorder (MDD) do not respond topsychopharmacological or psychotherapy treatments and some, who respond initially, may relapse and become unresponsive to subsequent treatment. Over half of people may not experience remission after the first or second antidepressant treatment course, and onethird may not experience remission after four different antidepressant treatment courses (Rush et al., 2006; Souery et al. 2007). Non-response rate to psychotherapy (typically cognitive behavioural therapy), has been reported at $70 \%$ (Griffiths \& Griffiths, 2014).

Depression is the leading cause of ill health and disability worldwide (WHO, 2017). Between $12 \%$ - $20 \%$ of depressed patients have TRD, and having TRD results in greater loss of economic contribution (Eaton et al., 2008; Nemeroff, 2007). Around $75 \%$ of the total costs related to depression are attributable to treatment resistant depression (TRD) (Mrazek et al., 2014).

In 2008 the US Food and Drug Administration (FDA) approved repetitive Transcranial Magnetic Stimulation (rTMS) for depression treatment (Janicak \& Dokucu, 2015). The UK's National Institute for Health and Care Excellence (NICE) (IPG 542, 2015) stated that it is safe and effective in reducing depressive symptoms compared to sham TMS, and it does not require hospital admission or anaesthesia (NICE, 2015). TMS neuromodulation employees an electromagnetic coil placed against the scalp to deliver a short, powerful magnetic field pulse to induce electric currents in the cerebral cortex (Hardy et al., 2016). Evidence indicates that rTMS changes brain activity, metabolism and connectivity that relate to emotional processing (Kito, Fujita, \& Koga, 2008); however, the exact mechanism of action is unknown (Hardy et al., 2016). rTMS is effective in treating the symptoms of depression (Griffiths et al., 2019; Health Quality Ontario, 2016).

Studies continue to prove the efficacy of ECT for treating severe depression (Mohn \& Rund, 2016; The UK ECT Review Group, 2003), with many showing ECT to be superior to pharmacotherapy (The UK ECT Review Group, 2003). ECT is still the most effective treatment for severe depression (Mohn \& Rund, 2016). ECT is associated with improvement in quality of life as assessed by both physicians and patients (Antunes \& Fleck, 2009).

Ketamine is an N-methyl-D-aspartate receptor antagonist (Andrade, 2018). Aligned with the glutamatergic hypothesis of depression (Sanacora et al., 2012), ketamine has been proposed as a treatment for depression (Hashimoto, 2009; Pittenger \& Duman, 2008; Tardito et al., 2006). When used to treat severe de- 
pression, evidence indicates that a subanaesthetic ketamine treatment dose provides a rapid and effective, but short term, antidepressant action (Kishimoto et al., 2016; Xu et al., 2015), might decrease suicidal ideation (Canuso et al., 2018) and cause a short term reduction in suicidality (Xu et al., 2015).

Ketamine achieves its maximum effect in 24 hours after administration and last up to seven days. Adverse effects include psychotic and dissociative effects, blood pressure and heart rate fluctuations, blurred vision and drowsiness (Grady et al., 2017). A meta-analysis concluded that ketamine has a rapid onset (within 1 day) antidepressant efficacy for those who have not benefitted from antidepressants, used as monotherapy or in combination with oral antidepressants (Fond et al., 2014). Another meta-analysis found ketamine exerts a large antidepressant effect in patients with MDD and bipolar disorder at 4 hours, 24 hours and 7 days (Coyle \& Laws, 2015). A review revealed seven RCTs of ketamine in MDD and bipolar disorder and it concluded that it has shown promise in quickly reducing depression symptoms in TRD (Grady et al., 2017). Ketamine dependence and withdrawal symptoms have been reported (Morgan et al., 2012).

In the US the FDA recently approved a ketamine nasal spray (SpravatoEsketamine [Canuso et al., 2018]) medication for treatment-resistant depression (FDA, 2019); and it is now available to treat depression in the USA. However, a nasal spray cannot deliver as precise a dose as an IV infusion (Gould et al., 2019). The UK's Royal College of Psychiatrists (2017) considers ketamine for depression a "novel treatment". A review and analysis of the ethical considerations in off-label ketamine use for treatment-resistant depression concluded it does not violate ethical principles, that guidelines for good practice should be enacted, and that the risk potential be monitored (Singh et al., 2017). In the UK intravenous ketamine is offered by two NHS healthcare providers to patients to treat depression as an "off-label" treatment.

Low doses of ketamine can increase motor cortex excitability to transcranial magnetic stimulation (Lazzaro et al., 2003). Very limited evidence has been reported on the combination of intravenous ketamine infusion and TMS treatments for depression. In one study subjects with major depressive disorder were pre-treated with ketamine or placebo prior to administration of theta burst stimulation (TBS). The study concluded that future research should examine the potential clinical impact of combining these treatments (Leuchter et al., 2017). A single case study of combined TMS/ketamine treatment for 12 weeks in TRD reported a significant reduction in depression but not remission (Best, 2015). Another single case study of TMS/ketamine combination therapy in TRD reported patient depression remission, which was sustained at the two-year point (Best \& Pavel, 2017). Best (2015, p. 3) stated: "We believe that modulation of the known dysfunctional cortico-thalamo-cortical circuit via the entraining effect of electromagnetic stimulation rendered the patient more responsive to the ketamine infusion. This resulted in more efficacious relief from the refractory depression... The result appears to be enduring, as the patient has been free of suf- 
fering for two years".

This paper reports analysis of routinely collected service data of patients who underwent intravenous ketamine infusion or both intravenous ketamine infusion and rTMS/ECT for TRD.

\section{Methods}

\subsection{Design}

The study was a retrospective investigation of routinely collected data on patients receiving ketamine infusions for depression between 2015 and 2019 at a UK based service provider. Demographic information (gender, age at admission), diagnosis, treatment funder, and outcomes data were extracted from clinical records containing routinely collected data. In this study, an anonymised database was created for analysis; an identity code was assigned to each patient in order to protect confidentiality so that no patient identifiers such as names, dates of birth or NHS numbers would appear on the database. The Head of Innovation and Research at Northamptonshire Healthcare NHS Foundation Trust responsible for research governance reviewed and approved the work.

\subsection{Measures}

The Beck Depression Inventory (BDI) is one of the most widely used selfreported measures of depression severity (Beck \& Alford, 2009). The scale has high content validity, construct validity, concurrent validities, content validity, internal consistency, and reliability (Jackson-Koku, 2016). The Clinical Global Impression (CGI) rating scale is one of the most widely used assessment instruments in psychiatry (Guy, 1976). The CGI is a brief clinician-rated instrument of illness severity. There is a lack of strong evidence for the validity and therefore it is recommended as part package of assessments (Forkmann et al., 2011). The 16-item Quick Inventory of Depressive Symptomatology (QIDS-SR) is a selfreported measure of depressive symptom severity, developed from the 30-item Inventory of Depressive Symptomatology (APA, 2000). The scale has high internal consistency and concurrent validity (Rush et al., 2006).

\subsection{Process to Treatment}

Patients with depressive symptoms are referred to the service by their GP or psychiatrist. A medical and physical history is taken, and patients are then assessed for a diagnosis of TRD by a psychiatrist working in the neuromodulation unit. TRD is defined as non-response to 2 or more appropriate courses of anti-depressants and a course of psychological therapy. Initial treatment of three ketamine infusions over a two to three week period to gauge the patient's response is undertaken; if patients respond further treatments are provided. rTMS protocol and length of treatment are set by the psychiatrist. Patients who lack capacity to consent to treatment are excluded. Patients are provided with information about the treatment (procedures, risks, side effects, remission/response 
rates) and are required to sign a "consent to treatment" form before treatment commences; they can withdraw consent at any point. rTMS equipment suppliers are Magstim and MagVenture.

\subsection{Intravenous Ketamine Infusion}

An anaesthetist inserts a needle into a vein on the back of patient's hand and sets up a syringe pump so that a dose of Ketamine is gradually infused over $40 \mathrm{~min}$ utes. Patients receive initial doses of $0.5 \mathrm{mg} / \mathrm{kg}$ this can be increased to $1 \mathrm{mg} / \mathrm{kg}$ depending on clinical response. The protocol is IV ketamine over 40 minutes for 3 test infusions to determine ketamine response, in those patients who do not exhibit a response after 3 ketamine infusions we discontinue ketamine and define the patient as a ketamine non-responder. In those patients who respond during the 3 test infusions will be told to return when symptoms come back or in 4 weeks whichever is the sooner.

\section{5. rTMS Treatment}

The site of stimulation is determined using the min- $\mathrm{R}$ "10 - 20" method with stimulation at the F3 electrode (Tsuzuki et al., 2016). The majority of patients received of FDA (Food and Drug Administration, 2019) depression protocol highfrequency stimulation to left dorsolateral prefrontal cortex and low-frequency inhibitory treatment to the right dorsolateral prefrontal cortex.

\subsection{ECT}

ECT delivery follows the NHFT (2019) protocol, which draws upon existing protocols. The Thymatron System IV ECT machine is used. A dosing algorithm based on seizure threshold (adapted from Fergusson et al. 2004) is used to determine the treating dose.

\subsection{Analysis}

Analysis was chosen with input from statistican. Analysis of change from baseline to the latest course treatment scores was carried out using data from 28 patients. Not all patients had data sets for all measures and so numbers per measure vary. For categorical responses with the DBI, we defined responses as a $50 \%$ or greater drop on the last assessment of treatment (Reeves et al., 2012), and $25 \%-49 \%$ drop as a partial response. Remission was defined as BDI score of $\leq 12$, CGI scores of $\leq 2$ (Riedal et al., 2010), and QIDS scores of $<5$ (Grammer et al., 2015).

As continuous variables were not normally distributed, Wilcoxon signed-rank tests $(Z)$ were used to compare baseline with post-treatment measures, together with the calculated effect sizes. Using non-parametric analysis (Spearman correlation coefficient), the relationship between the depression scales and ketamine treatment were explored. All tests were 2-sided, at $1 \%$ level of statistical significance. Data were analysed using statistics software package SPSS. 


\section{Results}

\subsection{Patient Characteristics}

The data were collected on a sample of 28 patients with TRD, who were treated between June 2015 and July 2019. The participants' age ranged from 22 to 78 years, with a mean of $49.41(S D=15.207)$. Women comprised $68 \%$ of the sample; however, crosstabulation indicated they were not overrepresented, $\chi^{2}$ ( $d f=1$, $n=28)=3.571, p=0.059$.

\subsection{Ketamine Treatment Outcome}

Baseline depression scores were in the severe range (see Table 1). There was a statistically significant improvement on BDI measures after the ketamine treatment, with a medium effect size. There was not a significant improvement in CGI or QIDS-SR.

\subsection{Categorical Response and Remission Rates}

BDI scores showed $23.1 \%$ responded to ketamine treatment, with a further $19.2 \%$ reaching a partial response. $57.7 \%$ of patients reported no response, with $4.17 \%$ reaching remission.CGI scores showed $18.18 \%$ responded to ketamine treatment, with a further $31.82 \%$ reaching a partial response. $50 \%$ of patients reported no response, with $4.55 \%$ reaching remission. QIDS scores showed 9.10\% responded to ketamine treatment, with a further $9.10 \%$ reaching a partial response. $81.82 \%$ of patients reported no response, no one reached remission.

\subsection{Clinician Assessed (CGI) and Self-Reported Measures (BDI/QIDS) of Depression}

There were no correlations found between the pre-treatment measures of depression, but there was a strong positive correlation between the two self-reported measures (CGI and BDI) at the last recorded day of ketamine treatment, $r=$ $0.71, p<0.001$.

\subsection{Impact of Other Variables}

There were no differences between BDI responders and non-responders in

Table 1. Mean (SD) pre- and post-treatment scores, mean change in scores and associated Wilcoxon Signed Ranks significance tests for patients treated with ketamine.

\begin{tabular}{cccccc}
\hline Rating Scale & $\boldsymbol{N}$ & Mean \pm SD [Range] & $\boldsymbol{Z}$ & $\boldsymbol{P}$ \\
\hline CGI & & & & & \\
Pre & 24 & $4.67 \pm 1.31[3-7]$ & -1.340 & 0.180 & 0.27 \\
Post & 25 & $4.12 \pm 1.13[2-7]$ & & & \\
BDI & & & & \\
Pre & 26 & $41.77 \pm 10.57[15-59]$ & -3.217 & $<0.001$ & 0.63 \\
Post & 26 & $32.04 \pm 13.67[1-52]$ & & & \\
QIDS-SR & & & & \\
Pre & 19 & $33.47 \pm 5.27[23-41]$ & -0.119 & 0.905 & 0.03 \\
Post & 20 & $31.95 \pm 7.53[13-44]$ & & & \\
\hline
\end{tabular}


gender $\left(\chi^{2}=163.0, p=0.529\right)$, age $(U=55.5, p=0.926)$ and the number of treatment sessions they received ( $U=58.0, p=0.929$ ). Similarly, no primary outcomes (age, gender, number of treatment sessions) were found to be statistically significant predictors of treatment effectiveness using binary logistic regression.

Apart from receiving ketamine treatment, some patients also received additional treatment (ECT or rTMS). Post-hoc ANOVA analysis (see Table 2) showed that for BDI, those with simultaneous ECT therapy scored the lowest scores, followed by those who received rTMS, and the highest depression scores were observed in those who only received ketamine treatment, suggesting ketamine works better when coupled with another form of therapy. Due to a lack of representative numbers, the gender differences between those who received rTMS at the same time as ketamine could not be calculated.

\section{Discussion}

The results show that a course of ketamine infusions (defined as a minimum of three) significantly improved depression on the DBI but not on the CGI or QIDS-SR. Response combined with partial response was above $42 \%$ on BDI and CGI and above $18 \%$ on the QIDS-SR; but remission rates for depression were less than $5 \%$ for all measures. The results, therefore, show a mixed picture of evidence of the impact ketamine on symptoms of depression in TRD over the course of multi infusions in clinical practice. The results need to be viewed in the context of clinical outcome data collection: outcome scores are taken prior to treatment and so are at a point when ketamine is not having an active effect.

The results may link to evidence indicating that a subanaesthetic ketamine treatment dose provides a rapid and effective, but short term, antidepressant action (Coyle \& Laws, 2015; Fond et al., 2014; Grady et al., 2017; Kishimoto et al., 2016; Xu et al., 2015). For those in this dataset who experienced remission or response, this may be due to short term relief allowing them to engage in activities (social, meaningful, physical, healthy lifestyle activities) that enabled a degree of mental health recovery. A qualitative investigation, interviewing patients who have undergone clinical ketamine treatment for depression would enable an understanding of how ketamine impacted on their lives and therefore provide a detailed insight into the nature of the effects of ketamine. It would allow an

Table 2. Multiple comparisons of BDI scores of patients who received ketamine only treatment or in conjunction with either ECT or rTMS.

\begin{tabular}{cccccccc}
\hline \multirow{2}{*}{$\begin{array}{c}\text { Dependent } \\
\text { Variable }\end{array}$} & $\begin{array}{c}\text { Additional } \\
\text { Treatment }\end{array}$ & $\begin{array}{c}\text { Additional } \\
\text { Treatment }\end{array}$ & $\begin{array}{c}\text { Mean } \\
\text { Difference }\end{array}$ & Std. Error & Sig. & \multicolumn{2}{c}{ 95\% Confidence Interval } \\
\hline \multirow{3}{*}{ BDI } & None & ECT & $16.44^{*}$ & 3.438 & $<0.001$ & 8.19 & 24.69 \\
& None & rTMS & $5.35^{*}$ & 1.102 & $<0.001$ & 2.71 & 8.00 \\
& rTMS & ECT & $11.09^{*}$ & 3.488 & 0.005 & 2.72 & 19.46 \\
\hline
\end{tabular}

${ }^{*}$ The mean difference is significant at the 0.05 level. 
understanding of the experience of the psychoactive ketamine effect and the subjective changes in mood, motivation and behaviour that resulted over time. It may also allow an investigation of any dependence and withdrawal symptoms.

There is likely to be an increase in availability and use of ketamine in the US following the FDA's recent approval of ketamine nasal spray (Spravato-esketamine) medication for treatment-resistant depression (FDA, 2019). In the UK it remains a "novel" treatment (Royal College of Psychiatrists, 2017) and it is uncertain if availability will expand beyond the two NHS healthcare providers treating depression with ketamine as an "off-label" treatment; or whether availability via private clinics will increase. Ketamine is a potential option to treat individuals with complex TRD when no further evidence-based therapies are available; nonetheless, more evidence on long-term efficacy and safety is required before inclusion in treatment algorithms of clinical practice guidelines (López-Díaz et al., 2019).

\subsection{Limitations}

Data were extracted from a clinical database and patient notes with some missing assessments, evidenced by the different number of subjects available for analysis on each outcome measure. Treatment was open label and adjunct to any existing antidepressant treatments, with the absence of a control. Data were from a single site in the UK limiting generalizability, however; patients were from across the UK, partially negating this. Ketamine effect trails off within 7 days.

\subsection{Conclusion}

More evidence is required about the longer term effects of ketamine for TRD. There is a need to effectively track the depression of people receiving ketamine at regular daily intervals following infusion; a smart phone based software application could enable this. A qualitative investigation of the experience of people receiving clinical ketamine for depression could provide insights into its effects and valuable information in relation to treatment decisions.

\section{Conflicts of Interest}

Professor Alex O'Neill-Kerr is a consultant to Magstim. No other authors have any conflicts of interest to declare.

\section{References}

Andrade, C. (2018). Ketamine as Anaesthesia for ECT: Is There Room to Improve a Gold Standard Treatment? The British Journal of Psychiatry, 212, 129-130. https://doi.org/10.1192/bjp.2017.15

Antunes, P. B., \& Fleck, M. P. (2009). Clinical Outcomes and Quality of Life in Patients Submitted to Electroconvulsive Therapy. The Journal of ECT, 25, 182-185. https://doi.org/10.1097/YCT.0b013e318190b2a9

APA (2000). Diagnostic and Statistical Manual of Mental Disorders (p. 4). Washington 
DC: American Psychiatric Association.

Beck, A. T., \& Alford, B. A. (2009). Depression: Causes and Treatment. Philadelphia, PA: University of Pennsylvania Press.

Berlim, M. T., \& Turecki, G. (2007). What Is the Meaning of Treatment Resistant/Refractory Major Depression (TRD)? A Systematic Review of Current Randomized Trials. European Neuropsychopharmacology, 17, 696-707. https://doi.org/10.1016/j.euroneuro.2007.03.009

Best, S. R. D. (2015). Combined Ketamine and Transcranial Magnetic Stimulation for Treatment Resistant Depression in the Context of Chronic OCD: A Case Report. Neuropsychiatric Electrophysiology, 1, 2. https://doi.org/10.1186/s40810-015-0003-y

Best, S., \& Pavel, D. G. (2017). Combined Transcranial Magnetic Stimulation and Ketamine for Treatment of Refractory Mood Disorder, Anxiety, and Pain: A Case Study. Current Neurobiology, 8, 1-4.

Canuso, C. M., Singh, J. B., Fedgchin, M., Alphs, L., Lane, R., Lim, P., Drevets, W. C. et al. (2018). Efficacy and Safety of Intranasal Esketamine for the Rapid Reduction of Symptoms of Depression and Suicidality in Patients at Imminent Risk for Suicide: Results of a Double-Blind, Randomized, Placebo-Controlled Study. American Journal of Psychiatry, 175, 620-630. https://doi.org/10.1176/appi.ajp.2018.17060720

Coyle, C. M., \& Laws, K. R. (2015). The Use of Ketamine as an Antidepressant: A Systematic Review and Meta-Analysis. Human Psychopharmacology: Clinical and Experimental, 30, 152-163. https://doi.org/10.1002/hup.2475

Eaton, W. W., Martins, S. S., Nestadt, G., Bienvenu, O. J., Clarke, D., \& Alexandre, P. (2008). The Burden of Mental Disorders. Epidemiologic Reviews, 30, 1-14. https://doi.org/10.1093/epirev/mxn011

FDA (2019). FDA Approves New Nasal Spray Medication for Treatment-Resistant Depression; Available Only at a Certified Doctor's Office or Clinic. Food and Drug Administration.

https://www.fda.gov/news-events/press-announcements/fda-approves-new-nasal-spray -medication-treatment-resistant-depression-available-only-certified

Fond, G., Loundou, A., Rabu, C., Macgregor, A., Lançon, C., Brittner, M., Roger, M. et al. (2014). Ketamine Administration in Depressive Disorders: A Systematic Review and Meta-Analysis. Psychopharmacology, 231, 3663-3676.

https://doi.org/10.1007/s00213-014-3664-5

Fergusson, G. M., Cullen, L. A., Freeman, C. P., \& Hendry, J. D. (2004). Electroconvulsive Therapy in Scottish Clinical Practice: A National Audit of Demographics, Standards, and Outcome. The Journal of ECT, 20, 166-173.

https://doi.org/10.1097/00124509-200409000-00008

Forkmann, T., Scherer, A., Boecker, M., Pawelzik, M., Jostes, R., \& Gauggel, S. (2011). The Clinical Global Impression Scale and the Influence of Patient or Staff Perspective on Outcome. BMC Psychiatry, 11, 83. https://doi.org/10.1186/1471-244X-11-83

Gould, T. D., Zarate Jr., C. A., \& Thompson, S. M. (2019). Molecular Pharmacology and Neurobiology of Rapid-Acting Antidepressants. Annual Review of Pharmacology and Toxicology, 59, 213-236. https://doi.org/10.1146/annurev-pharmtox-010617-052811

Grady, S. E., Marsh, T. A., Tenhouse, A., \& Klein, K. (2017). Ketamine for the Treatment of Major Depressive Disorder and Bipolar Depression: A Review of the Literature. Mental Health Clinician, 7, 16-23. https://doi.org/10.9740/mhc.2017.01.016

Grammer, G. G., Kuhle, A. R., Clark, C. C., Dretsch, M. N., Williams, K. A., \& Cole, J. T. (2015). Severity of Depression Predicts Remission Rates Using Transcranial Magnetic Stimulation. Frontiers in Psychiatry, 6, 114. https://doi.org/10.3389/fpsyt.2015.00114 
Griffiths, C. A., \& Griffiths, L. J. (2014). Recovery and Reliable Change Rates for Patients Scoring Severe on Depression, Anxiety or Impaired Functioning in a Psychological Therapies Service: IAPT. Mental Health Review Journal, 20, 28-35. https://doi.org/10.1108/MHRJ-06-2014-0022

Griffiths, C., O’Neill-Kerr, A., Millward, T., \& Da Silva, K. (2019). Repetitive Transcranial Magnetic Stimulation (rTMS) for Depression: Outcomes in a United Kingdom (UK) Clinical Practice. International Journal of Psychiatry in Clinical Practice, 23, 122-127. https://doi.org/10.1080/13651501.2018.1562077

Guy, W. (1976). ECDEU Assessment Manual for Psychopharmacology (pp. 534-537). https://doi.org/10.1037/e591322011-001

Hardy, S., Bastick, L., O’Neill-Kerr, A., Sabesan, P., Lankappa, S., \& Palaniyappan, L. (2016). Transcranial Magnetic Stimulation in Clinical Practice. BJPsych Advances, 22, 373-379. https://doi.org/10.1192/apt.bp.115.015206

Hashimoto, K. (2009). Emerging Role of Glutamate in the Pathophysiology of Major Depressive Disorder. Brain Research Reviews, 61, 105-123. https://doi.org/10.1016/j.brainresrev.2009.05.005

Health Quality Ontario (2016). Repetitive Transcranial Magnetic Stimulation for Treatment-Resistant Depression: A Systematic Review and Meta-Analysis of Randomized Controlled Trials. Ontario Health Technology Assessment Series, 16, 1.

Jackson-Koku, G. (2016). Beck Depression Inventory. Occupational Medicine, 66, 174-175. https://doi.org/10.1093/occmed/kqv087

Janicak, P. G., \& Dokucu, M. E. (2015). Transcranial Magnetic Stimulation for the Treatment of Major Depression. Neuropsychiatric Disease and Treatment, 11, 1549. https://doi.org/10.2147/NDT.S67477

Kishimoto, T., Chawla, J. M., Hagi, K., Zarate, C. A., Kane, J. M., Bauer, M., \& Correll, C. U. (2016). Single-Dose Infusion Ketamine and Non-Ketamine N-methyl-d-aspartate Receptor Antagonists for Unipolar and Bipolar Depression: A Meta-Analysis of Efficacy, Safety and Time Trajectories. Psychological Medicine, 46, 1459-1472. https://doi.org/10.1017/S0033291716000064

Kito, S., Fujita, K., \& Koga, Y. (2008). Regional Cerebral Blood Flow Changes after Low-Frequency Transcranial Magnetic Stimulation of the Right Dorsolateral Prefrontal Cortex in Treatment-Resistant Depression. Neuropsychobiology, 58, 29-36. https://doi.org/10.1159/000154477

Lazzaro, V. D., Oliviero, A., Profice, P., Pennisi, M. A., Pilato, F., Zito, G., Tonali, P. A. et al. (2003). Ketamine Increases Human Motor Cortex Excitability to Transcranial Magnetic Stimulation. The Journal of Physiology, 547, 485-496. https://doi.org/10.1113/jphysiol.2002.030486

Leuchter, A. F., Espinoza, R., Suthana, N., Hunter, A., \& Cook, I. A. (2017). Synergistic Effects of Ketamine and Theta Burst Stimulation in the Treatment of Major Depressive Disorder (MDD). Brain Stimulation: Basic, Translational, and Clinical Research in Neuromodulation, 10, 492. https://doi.org/10.1016/j.brs.2017.01.438

López-Díaz, Á., Murillo-Izquierdo, M., \& Moreno-Mellado, E. (2019). Off-Label Use of Ketamine for Treatment-Resistant Depression in Clinical Practice: European Perspective. The British Journal of Psychiatry, 215, 447-448.

https://doi.org/10.1192/bjp.2019.102

Mohn, C., \& Rund, B. R. (2016). Significantly Improved Neurocognitive Function in Major Depressive Disorders 6 Weeks after ECT. Journal of Affective Disorders, 202, 10-15. https://doi.org/10.1016/j.jad.2016.03.062 
Morgan, C. J., Curran, H. V., \& Independent Scientific Committee on Drugs (ISCD) (2012). Ketamine Use: A Review. Addiction, 107, 27-38. https://doi.org/10.1111/j.1360-0443.2011.03576.x

Mrazek, D. A., Hornberger, J. C., Altar, C. A., \& Degtiar, I. (2014). A Review of the Clinical, Economic, and Societal Burden of Treatment-Resistant Depression: 1996-2013. Psychiatric Services, 65, 977-987. https://doi.org/10.1176/appi.ps.201300059

Nemeroff, C. B. (2007). Prevalence and Management of Treatment-Resistant Depression. Journal of Clinical Psychiatry, 68, 17.

NHFT (2019). Protocol for Electroconvulsive Therapy (ECT) Treatment Centre within Northamptonshire Healthcare Trust. https://www.nhft.nhs.uk/download.cfm?doc=docm93jijm4n1809

NICE (2015). Repetitive Transcranial Magnetic Stimulation for Depression. https://www.nice.org.uk/guidance/ipg542

Pittenger, C., \& Duman, R. S. (2008). Stress, Depression, and Neuroplasticity: A Convergence of Mechanisms. Neuropsychopharmacology, 33, 88. https://doi.org/10.1038/sj.npp.1301574

Reeves, G. M., Rohan, K. J., Langenberg, P., Snitker, S., \& Postolache, T. T. (2012). Validation of BDI-II Response and Remission Cut-Points for Assessment of Seasonal Affective Disorder Patients. Journal of Affective Disorders, 138, 123. https://doi.org/10.1016/j.jad.2011.12.003

Riedel, M., Möller, H. J., Obermeier, M., Schennach-Wolff, R., Bauer, M., Adli, M., Bender, W. et al. (2010). Response and Remission Criteria in Major Depression: A Validation of Current Practice. Journal of Psychiatric Research, 44, 1063-1068. https://doi.org/10.1016/j.jpsychires.2010.03.006

Royal College of Psychiatrists (2017). Statement on Ketamine to Treat Depression. London: Royal College of Psychiatrists, Committee on ECT and Related.

Rush, A. J., Trivedi, M. H., Wisniewski, S. R., Nierenberg, A. A., Stewart, J. W., Warden, D., McGrath, P. J. et al. (2006). Acute and Longer-Term Outcomes in Depressed Outpatients Requiring One or Several Treatment Steps: A STAR ${ }^{\star}$ D Report. American Journal of Psychiatry, 163, 1905-1917. https://doi.org/10.1176/ajp.2006.163.11.1905

Sanacora, G., Treccani, G., \& Popoli, M. (2012). Towards a Glutamate Hypothesis of Depression: An Emerging Frontier of Neuropsychopharmacology for Mood Disorders. Neuropharmacology, 62, 63-77. https://doi.org/10.1016/j.neuropharm.2011.07.036

Singh, I., Morgan, C., Curran, V., Nutt, D., Schlag, A., \& McShane, R. (2017). Ketamine Treatment for Depression: Opportunities for Clinical Innovation and Ethical Foresight. The Lancet Psychiatry, 4, 419-426. https://doi.org/10.1016/S2215-0366(17)30102-5

Souery, D., Oswald, P., Massat, I., Bailer, U., Bollen, J., Demyttenaere, K., Zohar, J. et al. (2007). Clinical Factors Associated with Treatment Resistance in Major Depressive Disorder: Results from a European Multicenter Study. Journal of Clinical Psychiatry, 68, 1062-1070. https://doi.org/10.4088/JCP.v68n0713

Tardito, D., Perez, J., Tiraboschi, E., Musazzi, L., Racagni, G., \& Popoli, M. (2006). Signaling Pathways Regulating Gene Expression, Neuroplasticity, and Neurotrophic Mechanisms in the Action of Antidepressants: A Critical Overview. Pharmacological Reviews, 58, 115-134. https://doi.org/10.1124/pr.58.1.7

Tsuzuki, D., Watanabe, H., Dan, I., \& Taga, G. (2016). MinR 10/20 System: Quantitative and Reproducible Cranial Landmark Setting Method for MRI Based on Minimum Initial Reference Points. Journal of Neuroscience Methods, 264, 86-93.

https://doi.org/10.1016/j.jneumeth.2016.02.024 
The UK ECT Review Group (2003). Efficacy and Safety of Electroconvulsive Therapy in Depressive Disorders: A Systematic Review and Meta-Analysis. The Lancet, 361, 799-808. https://doi.org/10.1016/S0140-6736(03)12705-5

WHO (2017). Depression Fact Sheet. http://www.who.int/mediacentre/factsheets/fs369/en

Xu, Y., Hackett, M., Carter, G., Loo, C., Gálvez, V., Glozier, N., Mitchell, P. B. et al. (2015). Effects of Low-Dose and Very Low-Dose Ketamine among Patients with Major Depression: A Systematic Review and Meta-Analysis. International Journal of Neuro psychopharmacology, 19, pyv124. https://doi.org/10.1093/ijnp/pyv124 